\title{
A Similarity Retrieval Method for Functional Magnetic Resonance Imaging (fMRI) Statistical Maps
}

\author{
R. F. Tungaraza ${ }^{a}$, J. Guan ${ }^{a}$, S. Rolfe ${ }^{c}$, I. Atmosukarto ${ }^{a}$, A. Poliakov ${ }^{d}$, \\ N. M. Kleinhans ${ }^{e}$, E. Aylward ${ }^{e}$, J. Ojemann $^{b}$, J. F. Brinkley ${ }^{d}$, and L. G. Shapiro ${ }^{a}$ \\ ${ }^{a}$ Computer Science and Engineering, Box 352350, U. of Washington, Seattle, WA, USA 98195; \\ ${ }^{b}$ Neurological Surgery, Children's Hospital, 4800 Sand Point Way, Seattle, WA, USA 98105; \\ ${ }^{c}$ Electrical Engineering, Box 352500, U. of Washington, Seattle, WA, USA 98195; \\ ${ }^{d}$ Biological Structure, Box 357420, U. of Washington, Seattle, WA, USA 98195; \\ ${ }^{e}$ Radiology, Box 375115, U. of Washington, Seattle, WA, USA 98195
}

\begin{abstract}
We propose a method for retrieving similar fMRI statistical images given a query fMRI image and a database of such images. Our method creates a feature vector for each spatially distinct region within each such image in the database. The similarity between two images is obtained by the summed minimum distance (SMD) of their constituent feature vectors. Results on a dataset of fMRI statistical images from experiments involving three distinct cognitive tasks suggest that this method is able to capture the differences in activation patterns due to differences in the cognitive tasks performed.
\end{abstract}

Keywords: Functional imaging, Pattern recognition

\section{DESCRIPTION OF PURPOSE}

Statistical analysis on fMRI data culminate with the generation of statistical maps (e.g. Z-scores, T-scores, or F-scores) for individual subjects and/or groups of subjects. Consider a scenario where a number of research groups have conducted different types of fMRI experiments. The groups use an fMRI analysis method such as $\mathrm{SPM}^{1}$ to identify regions in their subjects' brains that were significantly activated due to the experimental stimuli. At the end of this process each group deposits their fMRI raw data and the accompanying statistical maps into a joint database.

Another researcher wants to find out whether the fMRI activation patterns of a subject not currently in the database are similar to any existing activation patterns in the database. She wants to retrieve other subjects regardless of the experimental condition and/or disorder that could potentially exhibit similar activation patterns. She also wants a numeric representation of the degree of similarity between the query image and all the retrieved images. This researcher may have set out to discover hidden similarities among different studies, identify similarities between datasets with a not-well-defined stimulus, or find similarities in brain activity when the cognitive tasks do not seem to be related based on psychological reasoning alone.$^{2}$

Besides those potential uses of this tool, doctors with patients who respond differently to treatments for a specific disease might be able to use this tool to identify the best group to which a given patient should be assigned and consequently administer the appropriate type of treatment. Suppose there are two distinct categories of brain activity for a given task following a stroke, each with a different treatment plan. Treatment A works best for patients in group A, and treatment B works best for those in group B. Our retrieval tool will enable such doctors to map a new patient with the same disorder into the best group and administer the appropriate treatment. These doctors will not only have a general activation pattern from each group to compare to, but also a score representing how similar/dissimilar the new patient's activation patterns are to every member of the two stroke-patients groups.

Further author information: (Send correspondence to R. F. Tungaraza)

R. F. Tungaraza: E-mail: rltungar@cs.washington.edu, Telephone: 12065435143

L. G. Shapiro: E-mail: shapiro@cs.washington.edu, Telephone: 12065432196 


\section{METHOD}

Ideally, the fMRI image analyst would have performed this step prior to using our tool. S/he would have used an fMRI image analysis method of their choice to apply a statistically significant threshold to all the statistical maps under analysis and generate their corresponding statistically significant clusters (spatially distinct regions).

We acquired a set of SPM t-contrast maps that spanned four distinct cognitive tasks: an auditory task, a memorization task, and two different face recognition tasks. These T-contrast maps were 3D images of the brain with each voxel representing the difference in the mean neuronal activation between two tasks performed by the same subject: task A versus task B (see Table 1). For system testing purposes, we restricted our analysis to those voxels exhibiting more activation for task A than for task B.

Preprocessing of the Images. We preprocessed each t-contrast map by removing all voxels with activation values less than or equal to zero. Among those voxels that were retained, we applied a threshold such that we retained the top X percent of activated voxels, where X ranged from 1 to 10 in steps of 1 . Then, we clustered the resulting voxels into spatially distinct regions. Note that the thresholding step in our process is artificial, and we test our procedures over multiple thresholds. In a full system, the data would have corresponding SPM T-maps, which would be compared to the T-statistics null distribution to determine the statistically significant activated regions within each image.

Table 1. Description of Experiments

\begin{tabular}{|l|l|l|}
\hline Experiment & Cognitive Process & Task A vs Task B \\
\hline $\begin{array}{l}\text { Auditory Oddball (AOD) } \\
(15 \text { subjects ) }\end{array}$ & $\begin{array}{l}\text { Recognize out } \\
\text { of place sound }\end{array}$ & $\begin{array}{l}\text { recognize a new tone vs } \\
\text { recognize the same repeating tone }\end{array}$ \\
\hline $\begin{array}{l}\text { Sternberg Working Memory (SB) } \\
\text { ( } 15 \text { subjects })\end{array}$ & $\begin{array}{l}\text { Recognize } \\
\text { memorized alphabets }\end{array}$ & $\begin{array}{l}\text { recognize memorized alphabets vs } \\
\text { recognize non-memorized alphabets }\end{array}$ \\
\hline $\begin{array}{l}\text { Face Recognition (Checkerboard) } \\
\text { ( } 12 \text { subjects })\end{array}$ & $\begin{array}{l}\text { Recognize } \\
\text { human faces }\end{array}$ & $\begin{array}{l}\text { recognize human faces vs } \\
\text { recognize a black \& white checkerboard }\end{array}$ \\
\hline $\begin{array}{l}\text { Face Recognition (Central-Cross) } \\
(24 \text { subjects })\end{array}$ & $\begin{array}{l}\text { Recognize } \\
\text { human faces }\end{array}$ & $\begin{array}{l}\text { recognize human faces vs recognize a black } \\
\text { cross at the center of a white background }^{5}\end{array}$ \\
\hline
\end{tabular}

To work around this, we preprocessed each t-contrast map by removing all voxels with activation values less than or equal to zero. Among those voxels that were retained, we applied a threshold such that we retained the top X percent of activated voxels. For our system X ranged from 1 to 10 in steps of 1 . Then, we clustered the resulting voxels into spatially distinct regions.

Feature Vector Creation. We transformed each spatially distinct region into a feature vector by extracting the following six properties: the region centroid, the region area, the average activation value for all the voxels within that region, the variance of those activation values, the average distance of each voxel within that region to the region's centroid, and the variance of the voxels distance to the region's centroid.

Similarity Measure. At this point, each brain contains a set of spatially distinct regions (or feature vectors) that are defined by the properties listed above. The similarity measure between a query brain and the other brains in the database is calculated by the Summed Minimum Distance (SMD) between the query brain Q and the target brain T. For every feature vector $\mathrm{s}$ in $\mathrm{Q}$ we calculate the Euclidean distance between s and every feature vector $\mathrm{r}$ in $\mathrm{T}$ and retain the minimum distance. Then we sum the minimum distances and divide the sum by the total number $N_{Q}$ of feature vectors in the query brain to obtain a query-to-target score. We perform the same procedure in the opposite direction to obtain a target-to-query score. The average of the query-to-target score and the target-to-query score is the SMD between the query and the target.

$$
\mathrm{SMD}=\frac{\text { Q-to-T Score }+ \text { T-to-Q Score }}{2}
$$




$$
\begin{aligned}
\text { Q-to-T Score } & =\frac{\sum_{r \in Q} \min _{s \in T} d(r, s)}{N_{Q}} \\
\text { T-to-Q Score } & =\frac{\sum_{s \in T} \min _{r \in Q} d(s, r)}{N_{T}}
\end{aligned}
$$

User Interface. Our system provides a graphical user interface that allows users to select query fMRI images and view the results as a subset of the slices of the most similar fMRI images in the database. The system can also be run in batch mode for evaluation purposes.

\section{RESULTS}

Evaluation. We evaluated the retrieval performance of our method in two ways. First, we calculated a random effects model (RFX) for each group. An RFX model represents a mean contrast map for a given group. If the query is an RFX contrast map from a given group, then the majority of target contrast maps should come from the same group as that of the query. Second, we used each individual 3D image in each group as a query image. In both sets of experiments we utilized the retrieval score ${ }^{6}$ below to score the retrieval results:

$$
\text { Retrieval Score }=\frac{1}{N \times N_{\text {rel }}}\left(\sum_{i=1}^{N_{\text {rel }}} R_{i}-\frac{N_{r e l}\left(N_{\text {rel }}+1\right)}{2}\right)
$$

$\mathrm{N}$ is the total number of brains in the dataset, $N_{\text {rel }}$ is the total number of brains within the query's group, and $R_{i}$ is the rank at which the $i^{t h}$ relevant brain is retrieved. A perfect retrieval where all the relevant brains are retrieved before any others would receive a score of 0 , while the worst retrieval where all the relevant brains are retrieved after all the others would receive a score of 1 . In the case of random retrieval, the score is approximately 0.5 .

RFX Model Retrieval. Figure 1(a) shows the changes in retrieval score when each of the RFX models was used as a query. The RFX model of the AOD, the SB, and the Checkerboard group had retrieval scores close to 0, while the Central-Cross group had near random retrieval scores (i.e. close to 0.5). The Central-Cross RFX models activation patterns are a subset of the activation patterns in the Checkerboard RFX model. During retrieval its top targets came from both the Central-Cross and Checkerboard group, which negatively affected its retrieval score.

Individual Brain Queries. Figure 1(b) shows the changes in average retrieval score when the individual fMRI images rather than the RFX model are used as queries. The only graph that exhibits a marked difference in shape to its corresponding graph in Figure 1(a) is the AOD average group scores. As the threshold increases the retrieval score of this graph increases at a much faster rate than its RFX model in Figure 1(a). Through visual inspection of the t-contrast maps of each member of the AOD group and computing the variance of the retrieval scores for this group, we found that this group's overall spatial activation pattern differed among group members.

\section{NEW OR BREATHTHROUGH WORK TO BE PRESENTED}

We provide a method for fMRI image analysts to compute the similarity between fMRI statistical maps of different subjects, under either the same or different experimental conditions. The method is accompanied by a computer system that uses a query fMRI statistical image to retrieve similar fMRI statistical images from a database. A graphical user interface allows users to select query fMRI images and view results. Figure 2 illustrates the results for one of the RFX model queries in our experiments.

\section{CONCLUSION}

We proposed and evaluated a method for retrieving similar fMRI statistical images given a query fMRI statistical image and a database of such images. The method is sensitive to similarities in brain activation patterns among members of the same experimental condition. In the future, we plan to test this method on datasets that do 

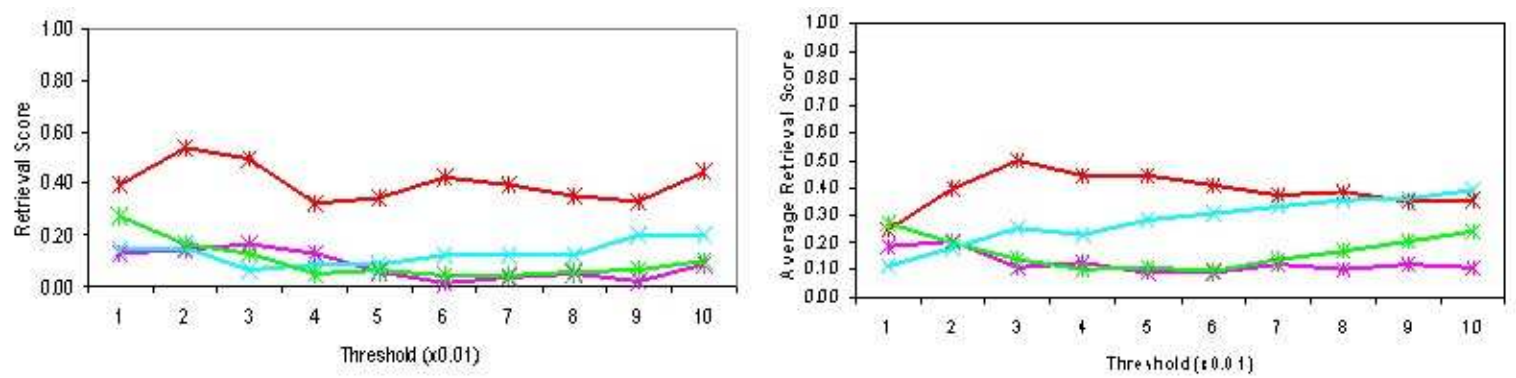

Figure 1. (a) Retrieved scores for the four RFX models (b) The average retrieval scores for each group over the individual query images in that group. Red: Central-Cross; Pink: Checkerboard; Green: SB; Turquoise: AOD.

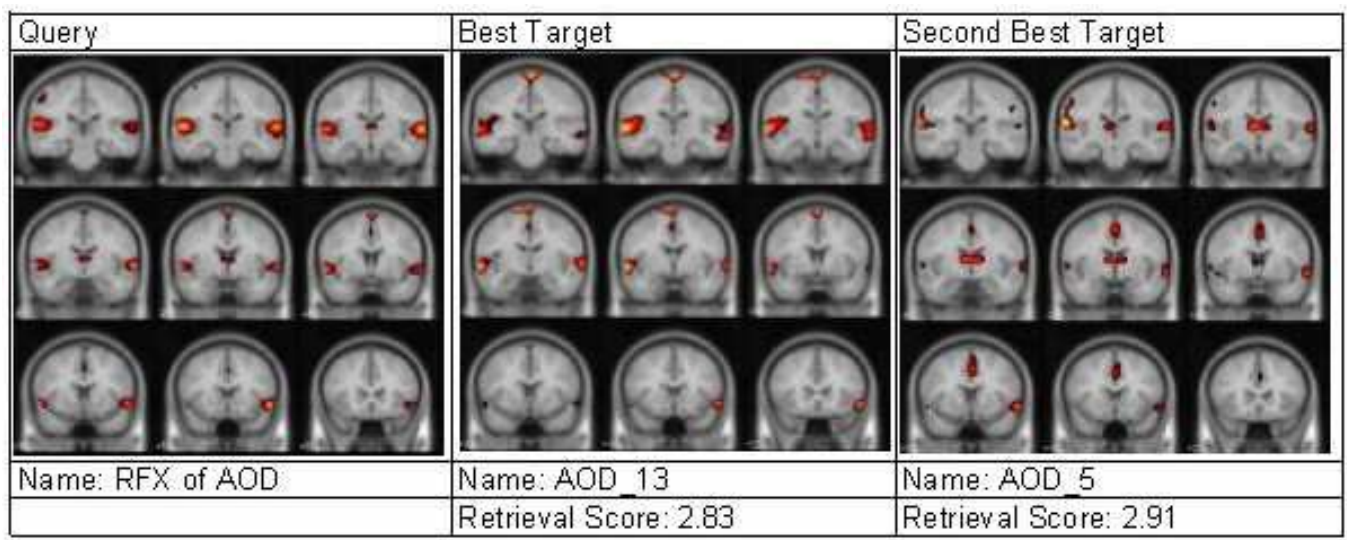

Figure 2. Top two retrieved 3D images using the RFX model of AOD as the query

not have a well-known brain activation pattern in an effort to aid scientific discoveries. In conjunction with collaborating brain researchers, we plan to use the method to study differences between groups affected with particular maladies, such as autism, and control groups. Lastly, we want to extend this method so it can be used to cluster statistical images from a set of subjects under the same experimental conditions into distinct groups based on their activation pattern similarities.

This work has not been submitted for publication or presentation elsewhere.

\section{REFERENCES}

[1] Friston, K., Ashburner, J., Kiebel, S., Nichols, T., and Penny, W., eds., [Statistical Parametric Mapping: The Analysis of Functional Brain Images], Academic Press (2007).

[2] Bai, B., Feature extraction and matching in content-based retrieval of functional Magnetic Resonance Images (fMRI), PhD thesis, Rutgers University (2007).

[3] Calhoun, V. D., Adali, T., Kiehl, K. A., Astur, R., Pekar, J. J., and Pearlson, G. D., "A method for multitask fmri data fusion applied to schizophrenia," Human Brain Mapping 27, 1-13 (2006).

[4] Henson, R., Shallice, T., Gorno-Tempini, M., and Dolan, R., "Face repetition effects in implicit and explicit memory tests as measured by fMRI," Cerebral Cortex 12, 178-186 (2002).

[5] Corrigan, N. V., Richards, T., Webb, S. J., Merkle, K., Kleinhans, N., Murias, M., Poliakov, A. V., Aylward, E., and Dawson, G., "An investigation into the relationship between fmri and erp source localized measurements of brain activity during face processing," In Press (2008).

[6] Muller, H., Marchand-Maillet, S., and Pun, T., "The truth about corel-evaluation in image retrieval," Proc. International Conference on Image and Video Retrieval 2383, 38-49 (2002). 